\title{
Family-Based Replication Study of Schizophrenia Genes
}

\author{
Karolina A. Aberg, PhD, Edwin J. C. G. van den Oord, PhD \\ Center for Biomarker Research and Personalized Medicine, Virginia Commonwealth University, \\ Richmond.
}

To the Editor In response to our family-based replication study of schizophrenia genes, ${ }^{1}$ Lazzeroni ${ }^{2}$ expressed concerns regarding the interpretation of the replication results and argued that simpler studies may ultimately yield faster progress in psychiatric genetics.

We presented 4 arguments that speak to the replication status of our results: (1) the highly significant enrichment of single-nucleotide polymorphisms (SNPs) with small $P$ values in the replication; (2) an $89 \%$ to $93 \%$ agreement between the direction of effects in our casecontrol genome-wide association study meta-analysis used to select SNPs and the SNPs that replicated; (3) several findings that replicated across ancestral groups; and (4) replicating SNPs were organized in meaningful biological patterns. Lazzeroni ${ }^{2}$ focused mainly on the second argument. Based on a simulation study involving 800 unrelated cases and control individuals, she suggested that odds ratios greater than 1 may be enriched among the top results (even if none of the SNPs have an effect) and that this artifact could potentially have caused the high agreement in our study. On behalf of the Family-Based Replication Consortium, we have 2 responses.

1. The simulations performed have no direct relation to our study. We performed logistic regression analyses in a sample of 11185 schizophrenia cases plus 10 768 control individuals to select more than 9000 SNPs that were replicated in 6298 individuals from 1811 nuclear families. The agreement measure we reported involved the concordance between the directions of effects in the genome-wide association study meta-analysis and the markers that replicated in our family-based replication study. Because of the obvious differences (eg, test performed, design, types of samples, and sample sizes) between our investigation and the simulation performed by Lazzeroni, ${ }^{2}$ we think that the extrapolation of findings from her simulation study is speculative.

2. The simulation study assumed a disease prevalence of $5 \%$ in the population, a minor allele frequency of 0.05 , and that study participants were randomly

Corresponding Author: Edwin J. C. G. van den Oord, PhD, Center for Biomarker Research and Personalized Medicine, Virginia Commonwealth University, 1112 E Clay St, McGuire Hall, Room 209B, Richmond, VA 23298-0581 (ejvandenoord@ vcu.edu).

Additional Information: The Family-Based Replication Consortium consists of Karolina A. Aberg, PhD; Youfang Liu, PhD; Jozsef Bukszar, PhD; Joseph L. McClay, PhD; Amit N. Khachane, PhD; Ole A. Andreassen, PhD; Douglas Blackwood, PhD; Aiden Corvin, $\mathrm{PhD}$; Srdjan Djurovic, PhD; Hugh Gurling, PhD; Roel Ophoff, PhD; Carlos N. Pato, MD; Michele T. Pato, MD; Brien Riley, PhD; Todd Webb, PhD; Kenneth Kendler, MD; Mick O’Donovan, PhD; Nick Craddock, PhD; George Kirov, PhD; Mike Owen, PhD; Dan Rujescu, PhD; David St Clair, PhD; Thomas Werge, PhD; Christina M. Hultman, PhD; Lynn E. Delisi, MD; Patrick Sullivan, MD; and Edwin J. van den Oord, PhD.

Conflict of Interest Disclosures: None reported. 
selected from the population. However, the median lifetime prevalence of schizophrenia is less than $1 \%,{ }^{3}$ a minor allele frequency of 0.05 is often the lower bound after quality control, and studies are more typically designed to have an approximately equal number of cases and control individuals (as we did for our study). When we replicated the simulations assuming an equal number of cases and control individuals, we did not observe the reported bias anymore and even under a random sampling scheme, it became very small, assuming minor allele frequency of 0.5 . Thus, it is unclear how relevant the author's basic observation is.

The use of a familial data set for the replication of findings from genome-wide association study meta-analyses did add complexity. However, we disagree with Lazzeroni that using statistical tools, such as a sign test, was overly complex. In genetics, most studies involve a case-control design that may introduce artificial differences. Although most biases can be controlled for, subtle artifacts can potentially remain (eg, population and ascertainment differences). When the sample sizes become increasingly larger, these artifacts can result in false discoveries. Because family-based studies are not susceptible to the same biases, they provide an opportunity to validate case-control findings, which, we believe, outweighs the disadvantage of the added complexity

\section{References}

1. Aberg KA, Liu Y, Bukszár J, et al. A comprehensive family-based replication study of schizophrenia genes. JAMA Psychiatry. 2013;70(6):573-581. [PubMed: 23894747]

2. Lazzeroni LC. Evaluating the evidence of replication for genetic associations with schizophrenia. JAMA Psychiatry. 2014;71(1):94-95.

3. Murray CJL, Lopez AD. The Global Burden of Disease. Geneva, Switzerland: World Health Organization; 1996. 